\title{
Research of Present Situation and Development Trend of Chinese Cosmetics Regulations
}

Zijin Zhang, Jun Zhu"

China Cosmetic Collaborative Innovation Center, Beijing Technology and Business University, Beijing, China

"Corresponding author: Jun Zhu, China Cosmetic Collaborative Innovation Center, Beijing Technology and Business University, No. 11/33, Fucheng Road, Haidian District, Beijing, China Tel.: +861068984945 Email: 13661302748@163.com

Received May 12, 2016

Revised August 10, 2016

Accepted August 24, 2016

Published September 30, 2016

\begin{abstract}
To promote further standardizing and scientific development of the Chinese cosmetics, trends of Chinese cosmetics regulations are analyzed. By investigating the current situation of cosmetic regulations, and comparing with foreign cosmetics laws and regulations, this paper expounds the existing problems of China's cosmetics regulations. China's regulatory system couldn't keep up with rapid development of cosmetics industry and the security supervision system of regulations is lagging. Lack of relative laws and regulations about efficacy evaluation sensory evaluation and unclear responsibilities between the managements restricted the development of cosmetic industry. It is suggested to improve the Chinese cosmetics legal system, strengthen supervision, and improve the management system.
\end{abstract}

Keywords: Cosmetics, Laws and regulations, Present situation, Development trend, China

\section{Introduction}

随着中国经济呈迅速上升的态势、科技日趋发达、社会越 来越进步，人们对物质文化和精神文化的需求也不断提升， 化妆品作为满足人们对物质生活的要求和丰富精神世界的 消费品，已经成为大众必不可少的生活日用品。改革开放 以来化妆品行业迅猛发展, 平均每年的复合增长率都有2位 数，据欧瑞国际统计，中国化妆品在2014年间的零售交易规 模达到2937 亿元，约占全球化妆品市场的 8.8\%，超越日本 成为全球第二大化妆品消费市场。目前中国化妆品相关法 律法规跟不上行业快节奏的发展, 一定程度上制约了国内化 妆品行业的发展进程。为了保证化妆品的质量, 保障消费者 的身体健康, 引导化妆品行业更加规范化、更具科学性的发 展, 进一步完善中国化妆品相关法律法规具有重要意义。

\section{The present situation and development trend of Chinese cosmetics regulations}

1. Major laws and regulations of China's cosmetics and related documents

20世纪80年代中期, 中国的化妆品法律法规体制基本形成, 经过不断的发展和完善, 目前中国已经形成了具有中国特色 的化妆品法律规范部门体系（Wang, 2013）。化妆品的监督 管理关系到化妆品的使用安全和消费者的健康, 最初中国的 化妆品监督管理工作主要由卫生部负责, 2008年9月开始化妆 品监督管理的职能移交给国家食品药品监督管理局。现在中 国对化妆品的监管主要由国家食品药品监管部门、质量监督 检验检疫部门和工商行政管理部门联合进行。国家食品药品 监督管理局主要负责对化妆品进行安全管理, 组织起草化妆 品监督管理的法律法规草案、拟订政策规划，协调化妆品安 全检测和评估工作, 组织展开对化妆品重大安全事故的调查 和处理。国家质量监督检验检疫总局依据《工业产品生产许 
可证管理条例》负责化妆品企业生产许可证的发放和监督管 理。国家工商行政管理局依据《消费者权益保护法》和《广 告法》对化妆品广告宣传和维护消费者权益方面进行监管。

目前中国化妆品监督管理法规体系主要包括法规、部门规 章、规范性文件和技术标准等部分。其中, 化妆品法规主要有 《化妆品卫生监督条例》, 涉及化妆品的重要部门规章和规范 性文件共有29部, 包括《化妆品卫生规范》、《化妆品生产企 业卫生规范》、《化妆品产品技术要求规范》等。

1) Implementing rules of cosmetic health supervision regulations

《化妆品卫生监督条例》是中国首部针对化妆品监督管理指 定的法规, 是中国一直以来实施化妆品监管的最主要的法律依 据。1989年9月26日国务院批准通过，由卫生部于同年11月13 日正式颁布了《化妆品卫生监督条例》, 标志着中国化妆品监 管法制化的开端（Yi, 2012），该条例规定定义化妆品的概念， 建立了化妆品生产企业卫生许可证制度, 将化妆品分为实施备 案制的普通化妆品和实施许可制的特殊化妆品, 对新原料进行 划界并规定其使用需经卫生部批准，制定化妆品标签和广告的 规范, 确立进口化妆品许可制, 规定化妆品卫生监督机构及其 职责，对某些违法行为制定处罚 (Song et al., 2015)。为完善 和细化《化妆品卫生监督条例》的相关法律规定，1991年3月 27 日卫生部发布《化妆品卫生监督条例实施细则》, 该细则详 细规定《化妆品生产企业卫生许可证》、特殊用途化妆品及进 口化妆品卫生的审核批准程序, 明确特殊用途化妆品的定义, 进一步明确化妆品卫生监督机构职责和分工。

国家食品药品监督管理总局于2014年11月8日发布的《化妆品 监督管理条例 (征求意见稿) 》是对《化妆品卫生监督条例》 的首次修订。《化妆品监督管理条例 (征求意见稿)》在化妆 品的定义中加入了牙齿及口腔秥膜产品, 意味着牙膏和漱口水 等口腔护理产品也将纳入化妆品管理范围。同时, 该条例规定 对互联网交易第三方平台实行生产经营者实名登记制度, 明确 第三方提供者的责任义务, 互联网交易是近年来兴起并已占有 重要地位的化妆品交易方式, 加强对互联网化妆品交易的关注 十分必要。2015年6月26日, 为加强化妆品监督管理, 保证化 妆品质量安全, 保障消费者的健康, 国务院法制办公室公布食 品药品监管总局起草的《化妆品监督管理条例（修订草案送审 稿）》并向社会各界公开征求意见。条例对化妆品生产经营者 做了清晰的定义, 将美容美发机构及宾馆等在经营服务中使用 化妆品或提供化妆品的企业或个人划归为化妆品经营者, 这些 机构是消费者接触化妆品的重要渠道, 明确化妆品经营者的范 围有助于使化妆品的经营更加规范化, 避免出现经营者损害消 费者利益、逃避责任的事件。
2) Hygienic standard for cosmetics

1987年, 卫生部发布了《化妆品卫生标准》是第一个关于 化妆品及其原料质量安全性评价的技术依据。1999年, 为 了完善和化妆品的相关技术标准, 卫生部参考欧盟化妆品 规程76/768/EEC制定并发布了《化妆品卫生规范》, 并于 2002年和 2007 分别对其进行了 2次修订。2007版的《化妆 品卫生规范》增加了对霉菌和酵母菌的检测方法, 调整禁用 原料、限用物质、限用防腐剂、限用着色剂和限用染发剂 成分, 将限用紫外线吸收剂更名为限用防晒剂, 增加了4中 限用防晒剂成分, 增加新的原料检测方法。《化妆品卫生规 范》（2007年版）实行约十年, 存在一些概念、术语和定义 的表述不够清晰, 有打印错误及书写翻译不规范的内容, 部 分检测与评价方法滞后或缺失, 部分化妆品原料、产品的安 全技术要求相对滞后等问题。

2015年8月12日, 国家食品药品监督管理局结合化妆品行 业发展和监管现状, 参考了关于化妆品方面的专业知识, 将《化妆品卫生规范》（2007年版）与全球主要国家与地 区相关法规标准比较分析, 对其进行修订, 形成了《化妆品 安全技术规范》（征求意见稿）。通过征求社会各界的意见 和建议, 对 《化妆品安全技术规范》（征求意见稿）进行逐 步修改和完善, 国家食品药品监督管理局于12月23日发布 2015年第268号令, 公布经化妆品标准专家委员会全体会议 审议通过, 自2016年12月1日起开始实施《化妆品安全技 术规范》(2015年版)。

《化妆品安全技术规范》相对《化妆品卫生规范》 （2007年版）有以下变化：对一系列化妆品名词术语进 行精准的解释; 规范成分的中文名称、英文名称及INCI名 称; 删除或修改与口腔卫生用品相关条款; 有关重金属及 安全性风险物质的风险评估标准更加严苛, 增加镉和二噁 烷的限量要求并规定不得检出石棉; 禁用原料从1286项增 加为 1388 项, 其中禁用植 (动) 物原料由 78 种变为 98 种; 调整限用组分、准用防腐剂、准用防晒剂、准用着色剂和 准用染发剂成分; 增加 60 个对禁限用物质的检验方法; 将粪大肠菌群修改为耐热大肠菌群; 规定防晒类化妆品中 二氧化钛和氧化锌的总使用量限制; 人体功效评价中增 加SPF标准品（P2和P3）的制备方法。本规范更加科学严 谨, 同时兼具先进性和规范性, 借鉴国际化妆品安全评价 的技术和经验, 充分结合中国化妆品的现状和科技发展的 程度, 进一步完善中国化妆品监管, 促进化妆品行业良性 发展。

3) Hygienic standard for cosmetics manufacturing enterprise

为加强对化妆品生产企业的卫生管理, 卫生部于1996年根 
据《化妆品卫生监督条例》及其实施细则制定并颁布了《化 妆品生产企业卫生规范》, 并于2000年7月5日 发布关于印发 《化妆品生产企业卫生规范》（2000年）的通知, 对《化妆 品生产企业卫生规范》(1996年)进行修订, 现行的《化妆品 生产企业卫生规范》（2007年）是第二次修订版，从2008年1 月1日起开始实施。《化妆品生产企业卫生规范》（2007年） 对化妆品原料及包装材料、生产企业的生产选址和建筑结 构、设施和设备的摆放安全、整个生产过程、成品贮存和出 入库的卫生要求制定规范, 规定了卫生管理部门的职责及对 化妆品从业人员资质的要求, 限制不符合条件的生产企业进 入市场, 合理约束生产企业的生产行为, 促进中国化妆品生 产企业的生产过程更加规范化。

4) Technical requirements for cosmetic products

化妆品产品技术要求是产品卫生质量安全的技术保障, 是 食品药品监督管理部门开展卫生监督执法的重要依据, 为了 更高效的对产品的卫生质量安全进行监管, 国家食品药品监 督管理于2010年11月26日制定了《化妆品产品技术要求规 范》（国食药监许〔2010〕454号），附件中发布了《国家食 品药品监督管理局化妆品产品技术要求》和《化妆品产品技 术要求编制指南》, 对化妆品产品技术要求的内容和格式进行 规范, 要求文件中必须包含产品名称、配方成分、生产工艺、 感官指标、卫生化学指标、微生物指标、检验方法、使用说 明、咜存条件、保质期等资料。对化妆品技术要求进行规范 化要求使化妆品卫生质量安全的监管更加便捷, 明确统一生产 企业需要上报的产品的具体信息, 加强中国化妆品技术规范。

\section{5) List of raw materials used in cosmetics}

《已使用化妆品原料名称目录》是中国各个化妆品生产企 业在生产经营化妆品时选择化妆品原料的重要参考依据。 中国已使用化妆品原料清单(2003版)中共有3265种原料, 其中包括一般化妆品原料2156种, 特殊化妆品原料（一般 限用物质、防腐剂、防晒剂、色素) 546种, 天然化妆品原 料（含中药）563种。2013年2月7日, 国家食品药品监管 总局发布的已批准使用的化妆品原料名称目录（第一批） 中包含原料1674种, 并且目录中录入了原料的中英文名称 和INCI名, 已批准使用的化妆品原料名称目录（第二批） 于同年5月10日发布, 包含了原料411种, 《已使用化妆品 原料名称目录(第三批)》(征求意见稿)中包含原料1356种。 2014年6月30日国家总局印发《关于已使用化妆品原料名 称目录的通告 (第11号) 》, 列出可使用原料8783种, 并 规定现在中国化妆品只能使用此目录中的原料, 否则就要 按新原料进行申报和审批。为进一步完善化妆品原料的管 理, 对已使用化妆品原料名录的制定和及时更新是有必要
的, 国家食品药品监管总局根据化妆品标准专家委员会意 见于2015年06月16日 发布通知, 拟对《已使用化妆品原料 名称目录》(2014年) 进行调整更新。现在科学技术飞速 发展, 化妆品原料多种多样, 规范化妆品中能使用的原料 对保障消费者健康起着重要的作用, 已使用化妆品原料名录 的制定和及时更新是有必要的。

\section{Current situations of Chinese cosmetic laws and regulations}

自改革开放以来，中国对化妆品行业予以高度重视，相关 部门陆续制定了多部法规、部门规章和规范性文件以及相 关标准, 同时也建立了化妆品市场监管的多个主要制度, 包括生产许可证制度、卫生许可证制度、化妆品不良反应 监测报告制度、普通化妆品备案制和特殊化妆品许可制、 进口化妆品许可制。虽然中国已经基本建立了规范化妆品 市场的法律框架, 但是中国目前化妆品监管制度还处于建构 和整合时期, 监管能力仍相对孱弱。随着社会和科技的不断 进步, 人们对生活品质的要求越来越高, 对化妆品安全的关 注和相关专业知识的认知逐渐提高, 同时由于化妆品种类繁 杂, 生产工艺及市场销售模式多样, 各个地区化妆品市场情 况复杂, 实际实行法律法规及相关规章制度时出现了很多问 题, 说明中国化妆品法律法规体系还有待完善。

1) Supervision of the system and the development of the industry are not coordinated

中国化妆品行业起步较晚, 但是发展速度非常迅猛, 化妆品 的种类不断翻新, 各个化妆品企业争放异彩, 化妆品生产模 式也逐渐从手工制作向更高效率的机械化发展, 市场经营方 式也有新的突破, 网上化妆品经营开始占据化妆品经营模式 的重要地位, 化妆品行业已经发展成为国民经济的支柱产业 之一。相较于化妆品行业的高速发展, 中国化妆品相关的法 律法规建设显得有些迟缓, 检测方法和检测手段不完善, 部 分的禁用物质和限用物质没够规范性标准, 从而无法出具权 威检测数据（Song et al., 2015），在化妆品安全风险检测、 缺陷产品的处理与召回、安全事故紧急控制措施和处理、有 关信息的发布与公开、产品功效评价等许多方面还存在缺陷

(Wang, 2013)。保持法律法规适应中国实际国情, 综合考虑 时代发展的规律, 制定与行业协调一致的监管体制, 有利于 化妆品行业更规范化、更好更快的发展。

2) Safety supervision system of laws and regulations is lagging

中国化妆品现在主要的法律依据还是1989年颁布的《化妆 品卫生监督条例》, 化妆品生产经营的社会环境已发生巨大 
改变, 安全监管法律体系相对滞后 $(\mathrm{Hu}, 2014)$ 。首先, 中国 现行化妆品法律法规体制过于依赖许可, 忽视对产品上市后 的安全监管, 化妆品不良反应的监测体系不健全, 由于规模 和地域的影响, 不能系统地规范化监管。上市后产品出现问 题, 主要靠医疗部门通过消费者使用化妆品以后出现皮肤或 其他不良反应就医后统计上报相关部门。由于消费者化妆品 不良反应的认知程度不同, 不去医疗机构诊治, 或发生问题的 案例比较分散, 产品安全问题不能及时被发现。同时, 化妆品 经营企业未实行经营许可管理, 对企业经营的动态信息不能 实时掌握, 产品出现缺陷或发现对身体有害的情况时不能第 一时间掌控产品的来源和去向, 有可能延误产品的召回和处 理, 导致安全事故波及范围的扩大, 不利于有效地进行监管。 另外，欧盟已经建立了由政府、企事业单位的多个领域的专 家组成的安全评价机构一消费者安全科学委员会 (scientific committee on consumer safety)。相比较而言, 由于中国安全 评价机制不够全面, 又缺乏长效性（Xu et al., 2015）, 比较重 视产品本身安全性, 缺乏对产品作用于人体的安全性, 通常 是产品出现安全问题以后再由政府组织专家进行安全评价, 缺少应急机制, 不能及时控制不安全产品的危害。

3) Lack of cosmetic efficacy evaluation and sensory evaluation

中国缺少化妆品功效评价和感官评价相关的法律法规或统 一标准, 更重视化妆品的安全评价, 对功效评价和感官评价 缺乏规范化。目前中国化妆品功效评价相关内容在《化妆品 卫生规范》（2007年版）中只有3项, 包括防晒化妆品防晒指 数 (sun protection factor, SPF 值) 测定、防水性能测试以及 长波紫外线防护指数（protection factor of UVA, PFA 值）的 测定。功效也是化妆品的质量的一部分, 对于保湿、美白、 延缓衰老等在化妆品宣称中常见的功效, 而化妆品宣称的真 实性是应当遵循的最根本原则（Yao et al., 2014），中国并没 有一个统一的规范化评价标准, 这关乎到如何鉴定化妆品产 品宣称是否属实, 化妆品的功效主要由企业的科研部或其他 科研机构进行, 机构之间对于功效评价的认可度存在问题。 而在感官评价方面中国才刚刚起步, 感官评价基本由经验丰 富的从业人员进行, 人的感官易受很多自身和外界因素的影 响, 人与人之间对同一事物的感知和喜好也存在差异, 导致 评价的结果有很大的不确定性, 中国化妆品感官评价还有待 完善。

4) Lack of uniformity of legal and regulatory documents 中国关于化妆品的法律法规和其他相关文件非常多, 但都 只是涉及到一部分, 同时不同法律法规文件存在冲突现象, 规范内容重复、缺乏协调性和统一性, 造成实际实施过程中
产生矛盾, 严重影响化妆品监管工作的效能。首先，《消费 品使用说明化妆品通用标签》中的国家标准和国家质检总 局《化妆品标识管理规定》的标准都对化妆品标签标识有规 定。此外, 《化妆品监督管理条例（征求意见稿）》在化妆 品的定义中加入了牙齿及口腔秥膜产品, 而《化妆品安全技 术规范》却删除了许多与口腔卫生用品相关的条款。再有, 《中华人民共和国产品质量法》的规定对生产或者销售不符 合国家卫生标准的产品的, 监管部门可以处违法生产、销售 产品（包括己售出和未售出的产品）货值金额等值以上3倍 以下的罚款, 而根据《化妆品卫生监督条例》的规定 “生产 或者销售不符合国家《化妆品卫生标准》的化妆品的, 没收 产品及违法所得, 并且可以处违法所得 3 到 5 倍的罚款”。由 于许多法律法规在不同的时期、根据不同的立法需求制定 (Ma \& Qian, 2015), 内容相对缺乏系统性和统一性, 对化妆 品的监管造成很多麻烦。

5) Management department is numerous, the power and responsibility is not clear

中国担任化妆品监督管理职责的机构众多, 各个部门有自 己的体系, 不同部门之间职责分配没有整体性, 导致责权不 明, 职能重叠与管理真空并存 (Ma \& Qian, 2015) , 没有形 成一个系统的、全面的、综合的化妆品监管体系 (Shang, 2014），易造成资源浪费和逃避责任的现象发生，给监管执 法工作带来了困难, 严重影响了监管效率。国家质检总局负 责工业产品的监督管理, 制定工业产品国家标准, 给企业发 放生产许可证, 而国家食品药品监督管理局负责化妆品的监 督管理, 制定化妆品国家卫生标准, 发放化妆品生产卫生许 可证, 食品药品监管部门和工商部门同时对化妆品抽查工作 负责, 在各个部门履行自己的职责时会出现重复管理的状 况。同时, 由于各个部门之间缺乏有效的沟通, 信息交流不 畅, 可能导致发布的信息存在不一致（Liang, 2012）。此外, 在化妆品检验方面, 各个机构有自己的检验机构, 不同机构 之间互不认可, 会造成检验结果缺乏权威性, 重复检验浪费 资源。中国化妆品管理部门存在的问题, 一定程度上阻碍了 化妆品行业的发展。

\section{Status quo of EU cosmetic laws and regulations}

欧盟是世界第一大化妆品市场, 也是中国化妆品行业的重要贸 易伙伴。对欧盟化妆品法律法规的研究非常重要, 中国化妆品 法规中的许多要求规范都参考了欧盟化妆品的相关法规。

1) Legal system is more perfect and pay attention to enterprise self-discipline

《化妆品法规》是欧盟最新出台的关于化妆品的法规, 消除 
了欧盟各个成员国之间技术性法规的分歧, 统一了化妆品法 规及相关要求 (Xing et al., 2015)。该规范制定了针对化妆 品的全面法规标准, 详细的规定安全评估要求, 具体明确禁 限用原料、CMR物质及纳米材料的要求, 来自生产过程、储 存、包装迁移中的痕量禁用物质，还涵盖了相关动物实验; 同时明确责任人与经销商的责任与义务; 消费者权益及化妆 品标签要求; 周密的市场监管, 包括市场中的控制措施、不 良反应监测等; 相关行政规定及违反规定的处罚; 产品备案 制度。相较中国化妆品法律法规现状, 欧盟的化妆品法律法 规体系更为完善, 法律文件更全面、更具系统性和开放性, 关于化妆品的安全监管的技术标准、行政方面规定, 化妆品 标签规范、消费者权益及化妆品生产经营者责任义务全都遵 守一个法规文件, 避免相关部门执行工作时出现文件依据有 分歧的情况。

欧盟化妆品法规详细规定了经销商的责任义务, 同时要求 每一种化妆品都要求有一个责任人, 并规定了成为责任人的 资格, 要求责任人要承担化妆品的安全质量责任, 确保产品 符合法律规定, 应向政府提供产品的相关资料, 以保证政府 能随时掌握化妆品的产品来源和流向等全部信息, 为化妆品 安全监管提供高效的监管手段 (Wang, 2011a)。欧盟化妆品 法规强调企业对化妆品的安全负有直接责任, 政府更重视对 上市后产品的监管, 并制定相关法律对企业行为进行约束和 管理，行业协会起到联系企业和政府部门之间的桥梁作用。

2) Enlightenment to Chinese cosmetics regulations

相对欧盟化妆品法规每年都进行修订, 我国化妆品相关的 法规更新较为滞后, 政策法规和技术评价体系都需要更加 完善, 建议密切关注欧盟及其他发达国家的化妆品法律法 规及修订的动态变化, 从保护消费者利益的角度出发, 充分 结合我国实际情况, 科学的制定和修订相关法规和标准, 统 一和规范需要遵守的法规文件, 明确责任分配, 对一些亟需 完善的文件及时补充修订并发布实施。此外, 针对新原料 和新技术应予以重视, 在对新原料和新技术可能存在的风 险的检测指标还需要完善。立法上善待实验动物, 鼓励使 用动物 “替代实验” 研发化妆品, 符合人道主义, 还有利于 打破欧盟等国禁止使用动物实验化妆品的进出口限制（He et al., 2014）。

\section{The development trend of Chinese cosmetic laws and regulations}

中国法律法规的发展趋势朝着更加规范化、科学化发展, 完 善中国化妆品的安全监督管理法律法规, 解决现有法律法规 中存在的问题, 能够切实保障消费者的身体健康不受侵害, 也有助于化妆品行业的可持续性发展（Yao et al., 2014）。

\section{1) Changes in regulatory patterns}

中国化妆品监管模式的主要特点是 “行政许可, 政府监 管”。欧、美、日等发达国家对化妆品产品安全的监管以生 产企业自律为主, 政府的监管为辅, 更注重于上市后产品的 监管 (Zhang, 2011)。参照其化妆品监管模式, 中国的监管 模式也呈现出注重企业自身责任的趋势, 2015年发布的《化 妆品监督管理条例 (修订草案送审稿) 》(简称《条例》) 中 规定中国化妆品监管遵循行业自律原则、行业自律原则和社 会监督三大原则。化妆品生产经营者应自觉遵守国家颁布的 化妆品法律法规及相关规定; 化妆品行业协会是化妆品行业 中除法律之外有权威性的组织, 连接消费者和企业的第三方 组织, 起督促引导的作用, 协会应严格自律, 建立行业信用 服务机制, 约束会员自觉遵守行业规定, 提高行业公信力

（Li, 2015）; 社会组织和个人有权对化妆品生产经营进行监 督并积极维护自己的合法利益。

2) Adjust the cycle of amendments to the laws and regulations

《条例》中原料管理的相关规定 “化妆品原料目录需要调 整的”, 食品药品监督管理部门应“于每年年底前将更新 后的目录重新发布”, 化妆品原料目录的更新周期定为一 年, 此前的法律法规文件中没有提及过调整目录的时间。 国际上，1976年正式在EEC官方杂志发布的《欧盟化妆品规 程》, 以附录 II、III、IV、VI、VII的形式, 分别规定化妆品 禁用物质、限用物质、着色剂、防腐剂、紫外线吸收剂, 之 后的每年都要进行重新修订。结合中国化妆品行业发展的速 度和特点, 借鉴发达国家颁布的最新化妆品法规, 适时地对 中国化妆品法律法规进行修订和完善, 定期更新相关文件以 适应市场现状, 将是中国化妆品法律法规发展的重要趋势。

3) Strengthen the evaluation of the effectiveness of cosmetics

中国的法律法规制定过程中对化妆品功效评价的重视程度 越来越高。2007年版的《化妆品卫生规范》与2002年版相比 较增加防晒化妆品的防晒效果评价方法, 最新发布的《化妆 品安全技术规范》（2015年版）将人体安全性和功效评价检 验方法拆分为人体安全性检验和人体功效评价检验方法两部 分内容, 人体功效 SPF 评价检验方法中增加高 SPF 标准品的 制备方法。《条例》中规定化妆品的功效宣称需要有充分的 科学依据, 其依据可以是相关文献资料或者研究数据。由此 可见, 中国的法律法规加强了对化妆品功效评价的重视, 有 助于中国化妆品更规范化, 维护消费者的合法权益。

4) Information openness 
《条例》中规定食品药品监督管理部门应将调整的化妆品 原料目录及时向社会公布, 新原料的有关信息应在批准和备 案后10个工作日向社会公布，特殊化妆品注册或者普通化妆品 备案信息需向社会公布，同时化妆品国家标准可供公众免费查 阅, 对查证属实的重大违法行为, 食品药品监督管理部门依法 通过媒体公开曝光。《关于调整化妆品注册备案管理有关事宜 的通告》规定自2014年6月30日起，国产非特殊用途化妆品将 正式实行产品信息网上备案，而进口非特殊化妆品的评审周 期为20个工作日, 未收到食品药品监督管理部门不予备案通 知的即可进口销售，让评审周期变得更加公开和透明。

5) Strengthen the management of new raw materials of cosmetics

化妆品新原料的监管是影响整个产业发展 “基础” 的变 革, 国家加强对化妆品新原料的管理是可以预见的。由于 科技不断进步，基因工程（Wu et al., 2011）、纳米技术 (Long \& Xie, 2015) 、细胞融合技术(Wang, 2011b)等前沿生 物技术被运用到化妆品原料的制备中，中国现行的化妆品法 律法规已不能满足日常监管, 极易造成某类产品的监管空 白 (Jiang, 2011)，对新原料的重视有助于完善法律监管体 系。《条例》对新原料的定义进行修改, 由“在国内首次使 用于化妆品生产的天然或人工原料”变为“在国内首次使用 于化妆品的天然或者人工原料”，弥补了国内经营的化妆品 新原料的监管漏洞, 同时规定了申请的流程并要求3年内每 半年报告其使用和安全情况。

\section{6) Thinning legal liability}

《条例》根据违法行为情节严重程度按照刑事与行政责 任、民事责任与行政责任、严重违法行为、较严重违法行 为、一般违法行为五个等级明确规定违法行为相应责罚, 另 外根据虚假申报骗取许可、未按规定备案、违规聘用人员、 拒绝监督检查等具体违法行为详细做出处罚规定, 对委托生 产、集中交易市场、互联网第三方品台、广告、检验机构、 审评和不良反应监测机构等相关责任方的违法行为特别制定 处罚和处分条例。中国化妆品法规更加明确化妆品企业违法 经营行为所需要承担的法律责任, 对由于化妆品产品质量安 全问题而导致消费者身体健康受到损害的案件提供更清晰具 体的法律依据, 细化法律责任为保障消费者的合法权益提供 更有力的支撑。

7) Pay more attention to the management of products after sale

上市后的产品监管机制缺乏是中国化妆品监管亟待解决的 问题，新颁布的《条例》中能体现政府开始重视化妆品售
后的监督管理工作。首先, 在缺陷产品召回方面, 要求化 妆品生产者在发现化妆品存在质量缺陷时需主动召回并采 取相应补救措施; 化妆品经营者发现上述情形的应停止经 营并立即通知相关生产经营者、消费者及食品药品监督管 理部门, 生产者认为需要召回的经营者协助召回; 食品药 品监督管理部门在日常检查中发现产品缺陷的应责令化妆 品生产经营者召回或停止生产经营。此外, 第五十三条规 定国家实行不良反应监测制度, 化妆品生产经营者主动监 测、及时报告, 相关机构、社会组织和个人发现有关化妆 品不良反应的报告化妆品不良反应监测机构, 不良反应监 测机构负责资料收集、分析和评价, 并向食品药品监督管 理部门提出处理意见。同时, 对已发生不良反应情况严重 的化妆品制定了一系列紧急控制措施, 要求食品药品监督 管理部门制定相应的质量安全事故应急预案, 化妆品生产 经营者需积极配合, 立即停止生产经营并制定事故处置方 案。对产品售后监管的重视, 弥补了中国化妆品法律法规 的缺陷, 促进化妆品行业更加规范化。

\section{Conclusion}

本文阐述近年来中国化妆品法律法规现状，分析现存问 题, 解读最新版《化妆品监督管理条例 (修订草案送审 稿）》, 有助于探索中国化妆品法律法规的发展趋势, 为进 一步完善中国化妆品法律法规、加强安全监督管理提供参 考, 为促进中国化妆品行业更加规范化、更具科学性的发展 提供思路。

\section{References}

He Y, Liu YL, Liu QQ. The improvement of legislation of cosmetic testing on animals: a comparison of the European Union and China. China Development, 14: 40-44, 2014.

Hu D. The research of cosmetics safety index detection method. Master's Thesis, Zhejiang University, 2014.

Jiang KX. Cosmetic standards and regulatory situation in China. Detergent \& Cosmetics, 34: 38-42, 2011.

Li SY. Development status and strategy analysis of Chinese cosmetic industry. Modern Economic Information, 4: 394-396, 2015.

Long Y, Xie JB. Research of microcapsule technology and nano cosmetics. Standard \& Quality of Light Industry, 2: 
57-58, 2015.

Liang XJ. Situation of cosmetic supervision in China. Detergent \& Cosmetics, 35: 33-35, 2012.

Ma M, Qian K. Cosmetic claims under China's new regulatory trend. Detergent \& Cosmetics, 38: 47-50, 2015.

Song HL, Li H, Tian ZX. Institutional reform on cosmetic regulatory governance network in China. Law and Economy, 3: 15-28, 2015.

Shang Q. Research on the perfection of China's cosmetics safety supervision regulation. Master's Thesis, Fudan University, 2014.

Wang Y. The studies of cosmetics safety regulation law in China. Master's Thesis, Southwest University, 2013.

Wang Y. Comparative study on the standard system of cosmetics in China and EU. Master's Thesis, Chinese Center for Disease Control and Prevention, 2011a.

Wang LX. PEG induced Haematococcus pluvialis cell fusion and screening of mutant. Master's Thesis, Nanjing Agricultural University, 2011b.

Wu M, Xu ZZ, Sun Y, Wang G, Chen G. Advance in the study of recombination collagens and its applications in cosmetics. Detergent \& Cosmetics, 34: 19-23, 2011.

Xu H, Wang ZT, He WJ, Xie T. Discussion on drug supervision responsibility and present situation, strategies of China cosmetics administration. Pharmacy and Clinics of Chinese Materia Medica, 6: 92-94, 2015.

Xing SX, Su Z, Zuo TT, Wang GL. EU regulations on cosmetics and its enlightenment. Chinese Journal of Health Laboratory Technology, 25: 3214-3216, 2015.

Yao JC, Zeng LG, Lin XW, Hu L, Du J, Li YB, Rao J. Analysis of status quo and related strategies of cosmetics safety control system in China. China Pharmacy, 25: 775-777, 2014.

Yi LW. The research of China's cosmetics market regulation legal system. Master's Thesis, Central South University, 2012.

Zhang JJ. Regulations, supervision and challenge of China's cosmetics. Oral Care Industry, 21: 34-37, 2011. 


\section{中文摘要}

\section{中国化妆品法规现状及发展趋势}

\section{张子衿, 祝钧"}

北京工商大学 中国化妆品协同创新中心, 北京, 中国

为了促进中国化妆品进一步规范和科学发展的趋势，本文对中国的化妆品法规进行了分析。通过调查化妆品法规的现 状，与国外化妆品法律法规比较，阐述了中国化妆品法规存在的问题。中国的监管体系跟不上化妆品行业快速发展 和安全监督体系法规滞后，缺乏相关法律法规之间的功效评价和感官评价，不清楚管理责任，限制了化妆品行业的发 展。建议加强监督, 完善管理体系来改善中国化妆品的法律体系。

关键词: 化妆品, 法律法规, 现状, 发展趋势, 中国 


\section{국문초록}

\section{중국화장품 법규현황 및 발전추세}

장자금, 축균

북경공상대학 중국화장품공동혁신센터, 북경, 중국

중국화장품 규범과 과학발전의 추세를 진일보 촉진하기 위하여 본 논문에서는 중국화장품 법규에 대해 분석하였다. 중국화 장품 법규의 현황을 조사하고, 이를 국외화장품 법규와 비교하여 중국화장품 법규에 존재하는 문제점을 상세히 설명하였다. 현재 중국의 관리감독체계는 화장품산업의 신속한 발전에 대응하지 못하고 있으며 특히 안전감독체계법규는 관련 산업의 발 전 단계에 비해 많이 지체되어있다. 그리고 효능평가 및 관능평가와 연관된 법률규정의 부족 및 불분명한 관리책임은 화장품 산업의 발전을 저해하고 있다. 관리 감독을 강화하고, 관리체계를 공고히 하여 중국화장품 법률체계를 개선해야 할 것이다.

핵심어: 화장품, 법규, 현황, 발전추세, 중국 
